\title{
Yukawa-like confinement potential of a scalar particle in a Gödel-type spacetime with any $l$
}

\author{
Mahdi Eshghi ${ }^{1, \mathrm{a}}{ }_{\mathbb{D}}$, Majid Hamzavi ${ }^{2,3}$ \\ ${ }^{1}$ Department of Physics, Imam Hossein Comprehensive University, Tehran, Iran \\ 2 Physics Department, Zanjan University, Zanjan, Iran \\ ${ }^{3}$ Department of Mathematics and Statistics, University of Texas at El Paso, El Paso, TX, USA
}

Received: 6 February 2018 / Accepted: 10 June 2018 / Published online: 25 June 2018

(C) The Author(s) 2018

\begin{abstract}
In this paper, we investigate the behavior of a scalar particle within the Yukawa-like potential in a Gödeltype space-time for any $l$. The behavior of a spinless particle is analyzed in the presence of a topological defect with analytical solutions to the Klein-Gordon equation. By using the generalized series and Nikiforov-Uvarof (NU) methods, we deduce the energy eigenvalues and eigenfunctions. We have discussed on the obtained results.
\end{abstract}

\section{Introduction}

Physically space-time is a mathematical model. This model combines space and time into a single continuum. All physical quantities can be described by a space-time. In fact, we can mean a four-dimensional continuum having spatial and temporal coordinates. For example, Albert Einstein could describe the gravity of a space-time as a geometric property and linked with the geometric quantity "curvature" in general relativity. In space-times, topological defects and the curvature can be presented by the Riemann curvature tensor or Riemann-Christoffel tensor. Also, in quantum gravity, topological defects can be found in the global monopole [1], and the cosmic string [2-4]. In fact, a quantum system can be affected by the topology and local curvature of the spacetime in a gravitational field. In this regard, see the energy levels of a hydrogen atom placed in the gravitational fields of a cosmic string or a global monopole [5], and the correction of energy levels depends on the features of the specific space-time. Also, during the last decades, some authors have widely studied the influences of topological defects on the non-relativistic or relativistic quantum systems such as bound states of electrons and holes to a disclination [6], the influence of the screw dislocation on the energy levels of non-

\footnotetext{
a e-mail: eshgi54@gmail.com; m.eshghi@ semnan.ac.ir
}

relativistic electrons [7], screw dislocations driven growth of nanomaterials [8], topological defects space times [9], the Landau levels in the presence of topological defects $[10,11]$, the influence of Coulomb potential and quantum oscillator in conical spaces [12], exact solutions of the Klein-Gordon equation in cosmic string space-time $[13,14]$ and others $[15-$ 17].

In this discussion, the Gödel space-time is very important in understanding the nature of time, rotation and gravitation. Also, we know that the Kurt Gödel cosmological solution has been obtained by Gödel for the rotating body in 1949 [18]. This stationary solution was calculated by considering a cylindrically symmetric for the Einstein equations of general relativity. In fact, it has interesting properties in area of physic. Further, the most appealing property of the Gödel space-time is the existence of closed time-like curves, that makes time travel a theoretical possibility and has certified an enduring appeal in the model [19-21]. Moreover, the Gödel space-time is not a pragmatic model for our universe, but, in various gravity theories, it can be a significant theoretical laboratory for considering a range of general properties of the space-time structure. In compact regions, researches of quantum computation have presented that the presence of closed time-like curves (CTCs) in space-time provides for a physical model in this computation [22,23]. In string theories, a few of researches of the possibility of CTCs have been also investigated by the Gödel space-time [24-28].

Later, in this geometry, the physical properties of the presence of CTCs was investigated by Hawking [29]. Beside, Reboucas et al. instituted three classes of solutions that are explained by the properties as: (a) solutions where there are no CTCs; (b) solutions with a sequence of alternating causal regions and not causal regions; (c) solutions explained by only one non-causal region [30,31]. In 2004 and 2005, Barrow et al. analyzed the other properties such as the stability and dynamic of the Gödel model $[32,33]$. 
Summary, some of the authors have investigated the application of the Gödel space-time in matter. For example, Cavalcante et al, have studied the quantum dynamics of quasi-particles in a continuum limit investigating an explanation of fullerene in a spherical solution of the Gödel-type space-time with a topological defect [34]. Garcia et al. have analyzed Dirac fermions in Gödel-type background spacetimes with torsion [35] and also they investigated a rotating fullerene molecule and applied a geometric theory to explain the fullerene as a two-dimensional spherical space with topological defects submitted to a non-Abelian gauge field [36]. In other work, they studied the Weyl fermions in a family of Gödel-type geometries in Einstein general relativity [37].

However, the Gödel's model is a special case of homogeneous models of the universe in the 1960s $[38,39]$. The SomRaychaudhuri space-time is a particular case of the Gödeltype space-time. Namely, the particular solution of the Gödeltype space-time is known the Som-Raychaudhuri space-time (Gödel flat solution) with the cosmic string [14,40]. Several researchers have studied the physical properties of a series of backgrounds with the Som-Raychaudhuri spacetime. For example, Paiva et al. have investigated the properties of the rotating Som-Raychaudhuri homogeneous spacetime [41], Wang et al. have studied the relativistic quantum dynamics of a spinless particle in the Som-Raychaudhuri space-time under the influence of the gravitational field produced by a topology [42], Carvalho et al. have worked about the Klein-Gordon oscillator in a Som-Raychaudhuri space-time with a cosmic dispiration [14] and Vitoria et al. have investigated Linear confinement of a scalar particle in a Som-Raychaudhuri space-time [43]. Also, there are a series of backgrounds with the cosmic string in Kerr space-time [44], Schwarzschild space-time [45,46], Gödeltype space-time [40] and AdS space [47].

On the other hand, the Yukawa potential is known a screened Coulomb potential in particle and atomic physics. In the 1930s, Hideki Yukawa presented that such a potential obtain from the exchange of a scalar field such as the field of a massive boson [48]. The potential is monotone increasing in $r$ and it is negative, implying the force is attractive. A form of Yukawa potential has been earlier applied by Taseli in obtaining a generalized Laguerre basis for hydrogen-like systems [49]. Also, Kermode et al. have applied various forms of the Yukawa potential to calculate the effective range functions [50]. Napolitano et al. have presented the analysis of expanded stellar kinematics of elliptical galaxies where a Yukawa-like correction to the Newtonian gravitational potential derived from $f(R)$-gravity is investigated as an alternative to dark matter in cosmological models [51]. Recently, a new other form of the Yukawa-like have been used by Ikhdair et al. by solution of the Klein-Gordon equation for a particle and anti-particle [52].
In here, we use Yukawa-like potential as Yukawa plus exponential with constant potentials for analyzing a relativistic scalar particle in Gödel's flat space-time using the KleinGordon equation. However, this equation can be solved in flat, spherical and hyperbolic spaces under the various interactions.

In this work, we consider a Som-Raychaudhuri spacetime (Gödel's flat) in the presence of a topological defect, and then analyze the Yukawa-like confinement of a relativistic scalar particle. We calculate the energy eigenvalues and wave functions by using the generalized series method. We discuss on the obtained results with more details. Although, we try that write the Klein-Gordon equation in spherical and hyperbolic spaces.

\section{Theory and calculations}

Now, we will consider a scalar quantum particle dynamic in a Gödel-type space-time.

\subsection{Scalar particle in Som-Raychaudhuri space-time}

In here, we would like that work to the string theory analog of Gödel's space-time that the general Gödel-type metric $[14,30]$ can be given in polar coordinates $(t, \rho, \varphi, z)$ in the presence of cosmic string as below:

$$
\begin{aligned}
d s^{2}= & -\left(d t+\alpha \Omega \frac{\sinh ^{2} l \rho}{l^{2}} d \varphi\right)^{2}+\alpha^{2} \frac{\sinh ^{2} 2 l \rho}{4 l^{2}} d \varphi^{2} \\
& +d \rho^{2}+d z^{2}
\end{aligned}
$$

where $\rho \in[0,+\infty)$, in the coordinate system, $(t, z) \in$ $(-\infty, \infty)$, the angular variable $\varphi \in[0,2 \pi]$ and the parameter of $\alpha, 0<\alpha<1$, is given in terms of the linear mass density of the string, $\lambda$ as $\alpha=1-4 \lambda$, while $\Omega$ characterizes the vorticity of the space-time and $l$ is as $l=0, \pm 1, \pm 2, \ldots$.

In this section, we investigate the quantum dynamics of a relativistic scalar particle under the Yukawa-like potential in the Gödel-type space-time when $l \rightarrow 0$ of metric Eq. (1), which is also called as the Som-Raychaudhuri spacetime [53] with an angle deficit.

In the Som-Raychaudhuri space-time, the metric tensor is defined by using cylindrical coordinates with the cosmic string with the line element below (with $c=\hbar=1$ ):

$$
\begin{aligned}
d s^{2}= & g_{\mu \nu} d x^{i} d x^{j}=d \rho^{2}+\alpha^{2} \rho^{2} d \varphi^{2} \\
& -\left(d t+\alpha \Omega \rho^{2} d \varphi\right)^{2}+d z^{2} .
\end{aligned}
$$

In here, we choose the curvilinear coordinate system as the circular cylindrical coordinate system, $\left(x^{0}, x^{1}, x^{2}, x^{3}\right)=$ $(t, \rho, \varphi, z)$. If we lead $\alpha \rightarrow 1$, then, the line element, Eq. (2), can be turned to the form as without planar angle deficit. 
Also, $\alpha \rightarrow 1$ and $\Omega \rightarrow 1$, in which the Som-Raychaudhuri space-time will be turned to the flat Minkowski spacetime.

And, in this case, the matric tensor is obtained as below:

$$
g_{\mu \nu}=\left(\begin{array}{cccc}
-1 & 0 & -\alpha \Omega \rho^{2} & 0 \\
0 & 1 & 0 & 0 \\
-\alpha \Omega \rho^{2} & 0 & \alpha^{2} \rho^{2}\left(1-\Omega^{2} \rho^{2}\right) & 0 \\
0 & 0 & 0 & 1
\end{array}\right)
$$

and, the contravariant components of metric tensor, $g_{\mu \nu}$, is written as follows:

$g^{\mu \nu}=\left(\begin{array}{cccc}\Omega^{2} \rho^{2}-1 & 0 & -\Omega / \alpha & 0 \\ 0 & 1 & 0 & 0 \\ -\Omega / \alpha & 0 & 1 / \alpha^{2} \rho^{2} & 0 \\ 0 & 0 & 0 & 1\end{array}\right)$

and, we obtain the determinant of metric tensor as $g=$ $\operatorname{det}\left(g_{\mu \nu}\right)=-\alpha^{2} \rho^{2}$.

The Klein-Gordon equation with the Laplace-Beltrami, $\nabla^{2}=\frac{1}{\sqrt{-g}} \partial_{\mu}\left(\sqrt{-g} g^{\mu \nu} \partial_{\nu}\right)$, under the scalar potential is written in the Som-Raychaudhuri space-time

$\left[\frac{1}{\sqrt{-g}} \partial_{\mu}\left(\sqrt{-g} g^{\mu \nu} \partial_{\nu}\right)-(M+V(r))^{2}\right] \phi(\mathbf{r}, t)=0$,

where $M$ is the mass of free particle, $\partial_{\mu}, \partial_{\nu}$ are derivatives with respect to the cylindrical coordinates.

In here, we choose the potential model as the spatiallydependent Yukawa-like potential that is given as follows:

$V(\rho)=-V_{1}+V_{2} e^{-\mu \rho}+V_{3} \frac{e^{-2 \mu \rho}}{\rho}$,

where $V_{1}, V_{2}, V_{3}$, and $\mu \geq 0$ are constant parameters of the potential. If we set the values of $V_{1}$ and $V_{2}$ as zero, $V_{1}=V_{2}=$ 0 , then, the potential will be reduced as Yukawa potential.

After the substituting Eq. (7) in Ref. [42], using the Taylor's series expansion of the exponential functions to the first approximation and substituting it into Eq. (6), we have:

$$
\begin{aligned}
& \frac{\partial^{2} \Phi(\mathbf{r}, t)}{\partial \rho^{2}}-\frac{\partial^{2} \Phi(\mathbf{r}, t)}{\partial t^{2}}+\left(\frac{1}{\alpha \rho} \frac{\partial}{\partial \varphi}-\Omega \rho \frac{\partial}{\partial t}\right)^{2} \Phi(\mathbf{r}, t) \\
& +\frac{\partial^{2} \Phi(\mathbf{r}, t)}{\partial z^{2}}+\frac{1}{\rho} \frac{\partial \Phi(\mathbf{r}, t)}{\partial \rho} \\
& -\left(M+V_{1}-V_{2}+2 V_{3} \mu+V_{2} \mu \rho-\frac{V_{3}}{\rho}\right)^{2} \Phi(\mathbf{r}, t)=0 .
\end{aligned}
$$

Now, we choose the wave function as $\Phi(r, t)=e^{-i \varepsilon t}$ $e^{i l \varphi} e^{i p_{z} z} u(\rho)$, where $u(\rho)$ is the radial part of the wave function, $z$ (in the interval $[-\infty,+\infty]$ ) and the angular momentum operator, $p_{z}$, is as $p_{z}=$ const.
Substituting the selected wave function into Eq. (7), we have:

$$
\begin{aligned}
& \frac{\partial^{2} u(\rho)}{\partial \rho^{2}}+\frac{1}{\rho} \frac{\partial u(\rho)}{\partial \rho} \\
& +\left[\left(-\frac{l^{2}}{\alpha^{2}}+V_{3}^{2}\right) \frac{1}{\rho^{2}}+2\left(M+V_{1}+V_{2}+2 V_{3} \mu\right) V_{3} \frac{1}{\rho}\right. \\
& +\left(\varepsilon^{2}-\frac{2 l \varepsilon \Omega}{\alpha}+2 V_{2} V_{3} \mu-p_{z}^{2}\right. \\
& \left.-\left(M+V_{1}+V_{2}+2 V_{3} \mu\right)^{2}\right) \\
& -2 V_{2} \mu\left(M+V_{1}+V_{2}+2 V_{3} \mu\right) \rho \\
& \left.-\left(\varepsilon^{2} \Omega^{2}+V_{2}^{2} \mu^{2}\right) \rho^{2}\right] u(\rho)=0
\end{aligned}
$$

Now, the change of the dependent variable $u(\rho)=\frac{1}{\sqrt{\rho}} G(\rho)$, Eq. (8) can be transformed to the compact Liouville's normal form

$$
\left(\frac{d^{2}}{d \rho^{2}}-\sum_{i=-2}^{2} \delta_{i} \rho^{i}\right) G(\rho)=0,
$$

where

$$
\begin{aligned}
& \text { where } \\
& \begin{array}{c}
\delta_{-2}=\frac{l^{2}}{\alpha^{2}}-V_{3}^{2}-\frac{3}{4}, \delta_{-1}=-2\left(M+V_{1}+V_{2}+2 V_{3} \mu\right) V_{3}, \\
\delta_{0}=\varepsilon^{2}-\frac{2 l \varepsilon \Omega}{\alpha}+2 V_{2} V_{3} \mu-p_{z}^{2}-\left(M+V_{1}+V_{2}+2 V_{3} \mu\right)^{2}, \\
\delta_{1}=2 V_{2} \mu\left(M+V_{1}+V_{2}+2 V_{3} \mu\right), \quad \delta_{2}=\varepsilon^{2} \Omega^{2}+V_{2}^{2} \mu^{2},
\end{array}
\end{aligned}
$$

In this stage, to solve Eq. (9), we use the generalized series method by Eshghi et al. in Refs. [54,55], parameters $\delta_{0}, \delta_{1}, \delta_{2}, \delta_{-1}$ and $\delta_{-2}$ given in Eq. (10), and by a little algebraic calculation, we can calculate the eigenvalues equation as below:

$$
\begin{aligned}
& \left(\varepsilon^{2} \Omega^{2}+V_{2}^{2} \mu^{2}\right)\left\{\sqrt{\varepsilon^{2} \Omega^{2}+V_{2}^{2} \mu^{2}}(2 n+1\right. \\
& \left.+\frac{1}{2} \sqrt{1+\frac{l^{2}}{\alpha^{2}}-V_{3}^{2}-\frac{3}{4}}\right) \\
& +4\left(\varepsilon^{2}-\frac{2 l \varepsilon \Omega}{\alpha}+2 V_{2} V_{3} \mu-p_{z}^{2}\right. \\
& \left.\left.-\left(M+V_{1}+V_{2}+2 V_{3} \mu\right)^{2}\right)\right\} \\
& -4\left(M+V_{1}+V_{2}+2 V_{3} \mu\right)^{2} V_{2}^{2} \mu^{2}=0,
\end{aligned}
$$

where $n$ is an integer number. We observe the energy eigenvalues depend explicitly on the constant parameters of the potential, $V_{1}, V_{2}, V_{3}$, and $\mu$, the linear mass density of the string, $\lambda$, and also, the vorticity parameter $\Omega$. This quantity, $\Omega$, characterizes the global structure of the metric in a particular case of the Gödel-type space-time, namely, the SomRaychaudhuri space-time. If, we set $V_{1}=V_{2}=0$, then, then, the eigenvalues equation of scalar particle, Eq. (11), will 


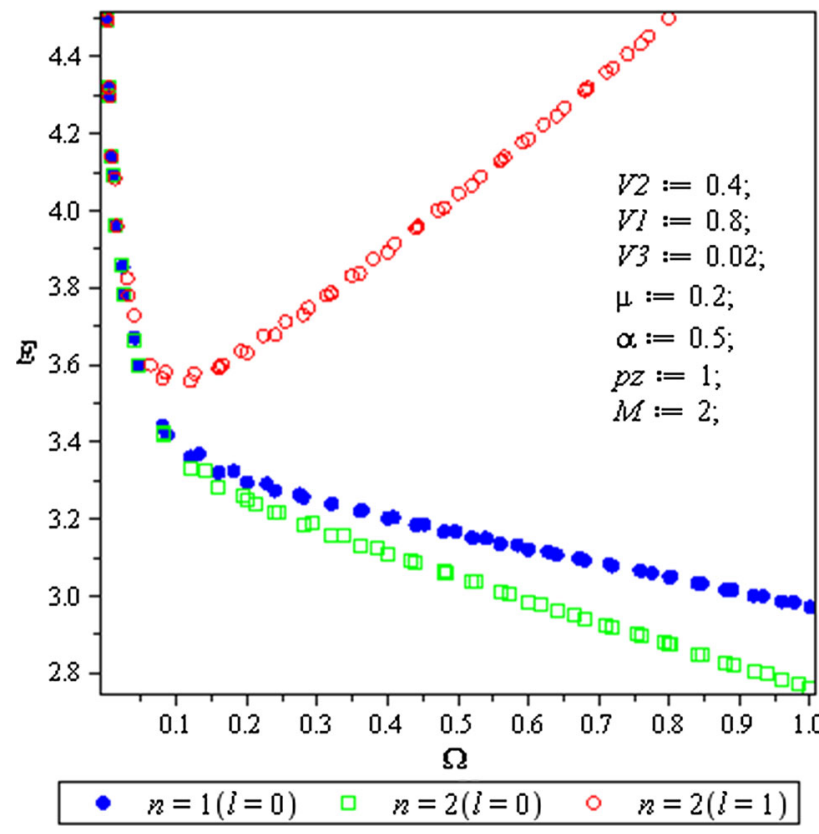

Fig. 1 Energy levels versus the vorticity parameter $\Omega$ with the different $n$ and $l$

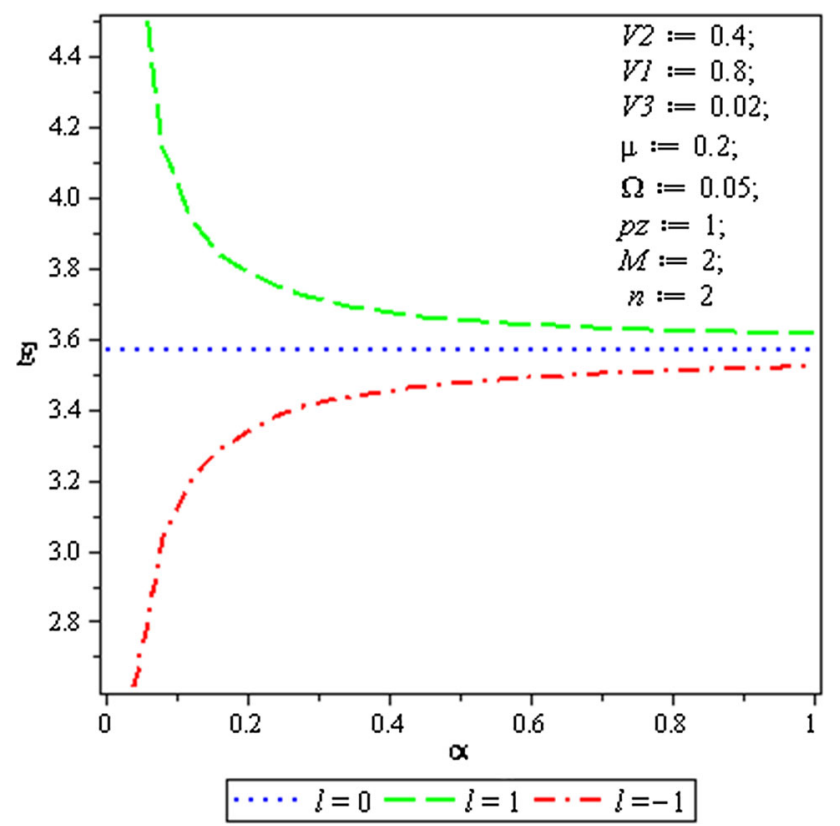

Fig. 2 Energy levels versus the parameter $\alpha$ with the different $l$

be transformed as $\varepsilon^{3} \Omega^{3}\left\{2 n+1+\frac{1}{2} \sqrt{1+\frac{l^{2}}{\alpha^{2}}-V_{3}^{2}-\frac{3}{4}}+\right.$ $\left.4\left(\varepsilon^{2}-\frac{2 l \varepsilon \Omega}{\alpha}-p_{z}^{2}-\left(M+2 V_{3} \mu\right)^{2}\right)\right\}=0$ for the Yukawa interaction.

In Figs. 1 and 2, we plot the energy levels versus the vorticity parameter $\Omega$ and the parameter of $\alpha$, respectively.
Finally, we can calculate the wave function as

$$
\begin{aligned}
& \Phi(\mathbf{r}, t)=e^{-i \varepsilon t} e^{i l \varphi} e^{i p_{z} z} \frac{1}{\sqrt{\rho}} \rho^{\frac{1}{2} \sqrt{1+\frac{l^{2}}{\alpha^{2}}-V_{3}^{2}-\frac{3}{4}}} \\
& \times \exp \left[\frac{1}{2} \sqrt{\varepsilon^{2} \Omega^{2}+V_{2}^{2} \mu^{2}}\right. \\
& \left.\left(\rho+\frac{2 V_{2} \mu\left(M+V_{1}+V_{2}+2 V_{3} \mu\right)}{2\left(\varepsilon^{2} \Omega^{2}+V_{2}^{2} \mu^{2}\right)}\right) \rho\right] D_{n+1}^{(3)}(\rho ; \mathbb{A}),
\end{aligned}
$$

with

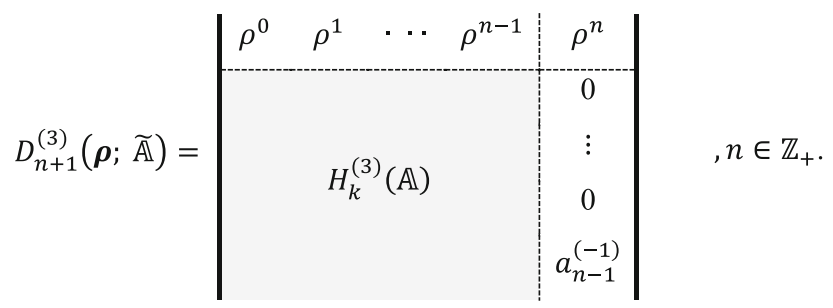

where

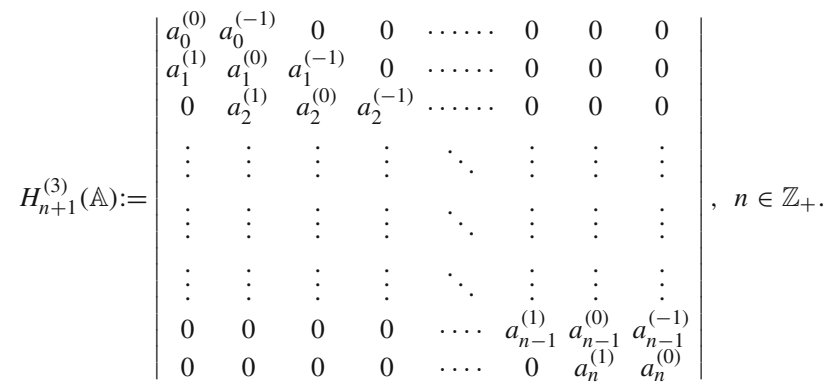

is known the Hessenberg determinant [56] of order $n+1$. In fact, the Hessenberg determinants help us this ability to obtain the solution of the problem explicitly and analytically. Using the Eq. (25) Ref. [55], the elements of the Hessenberg determinant are obtained as below:

$$
\begin{aligned}
& a_{i}^{(j)}:=(i+1)\left(i+1+\sqrt{1+4\left(\frac{l^{2}}{\alpha^{2}}-V_{3}^{2}-\frac{3}{4}\right)}\right), \\
& a_{i}^{(j)}:=i-2 V_{2} \mu\left(M+V_{1}+V_{2}+2 V_{3} \mu\right)
\end{aligned}
$$

$$
-\frac{V_{2} \mu\left(M+V_{1}+V_{2}+2 V_{3} \mu\right)}{\sqrt{\varepsilon^{2} \Omega^{2}+V_{2}^{2} \mu^{2}}}
$$

$$
\left(3+\sqrt{1+4\left(\frac{l^{2}}{\alpha^{2}}-V_{3}^{2}-\frac{3}{4}\right)}\right),
$$

$a_{i}^{(j)}:=\frac{\left(M+V_{1}+V_{2}+2 V_{3} \mu\right)^{2} V_{2}^{2} \mu^{2}}{\varepsilon^{2} \Omega^{2}+V_{2}^{2} \mu^{2}}$ 


$$
\begin{aligned}
& -\varepsilon^{2}-\frac{2 l \varepsilon \Omega}{\alpha}+2 V_{2} V_{3} \mu-p_{z}^{2} \\
& -\left(M+V_{1}+V_{2}+2 V_{3} \mu\right)^{2} \\
& -\sqrt{\varepsilon^{2} \Omega^{2}+V_{2}^{2} \mu^{2}}(4(i-1)+2 \\
& \left.+\sqrt{1+4\left(\frac{l^{2}}{\alpha^{2}}-V_{3}^{2}-\frac{3}{4}\right)}\right),
\end{aligned}
$$

where $j=-1,0,1$ and $a_{i}^{(j)}=0$ for $i<0$.

\subsection{Scalar particle in spherical symmetrical Gödel space-time}

Now, we investigate the limit of Eq. (1) where we may calculate a class of solutions of spherical symmetrical Gödel-type space-time by substituting $l^{2}<0$ in Eq. (1). Therefore, the metric tensor can be written in the presence of the cosmic string as below [49]:

$$
\begin{aligned}
d s^{2}= & \frac{4 R^{2}}{4 R^{2}+\rho^{2}}\left(d \rho^{2}+\alpha^{2} \rho^{4} d \varphi^{2}\right) \\
& -\left(d t+\frac{4 R^{2} \alpha \Omega}{4 R^{2}+\rho^{2}} \rho^{2} d \varphi\right)^{2}+d z^{2} .
\end{aligned}
$$

In here, the matric tensor is as below:

$$
g_{\mu \nu}=\left(\begin{array}{cccc}
-1 & 0 & \frac{\left(\alpha \Omega 4 R^{2}+1\right) \rho^{2}}{4 R^{2}} & 0 \\
0 & \frac{\left(4 R^{2}+\rho^{2}\right)^{2}}{16 R^{4}} & 0 & 0 \\
\frac{\left(\alpha \Omega 4 R^{2}+1\right) \rho^{2}}{4 R^{2}} & 0 & \frac{\alpha^{2} \rho^{4}\left(1-\Omega^{2}\right)\left(4 R^{2}+\rho^{2}\right)^{2}}{16 R^{4}} & \\
0 & 0 & 0 & 0 \\
& & & 1
\end{array}\right),
$$

and the inverse matric is obtained as follows:

$$
g^{\mu \nu}=\left(\begin{array}{cccc}
\Omega^{2}-1 & 0 & -\left(1+\frac{\rho^{2}}{4 R^{2}}\right) \frac{\Omega}{\alpha \rho^{2}} & 0 \\
0 & \frac{16 R^{4}}{\left(4 R^{2}+\rho^{2}\right)^{2}} & 0 & 0 \\
\left(1+\frac{\rho^{2}}{4 R^{2}}\right) \frac{\Omega}{\alpha \rho^{2}} & 0 & \frac{16 R^{4}}{\left(4 R^{2}+\rho^{2}\right)^{2} \alpha^{2} \rho^{4}} & 0 \\
0 & 0 & 0 & 1
\end{array}\right) .
$$

And $g=\operatorname{det}\left(g_{\mu \nu}\right)=-\frac{\alpha^{2} \rho^{4}\left(4 R^{2}+\rho^{2}\right)^{4}}{256 R^{8}}$.

Therefore, in this space, the Klein-Gordon equation under the Yukawa-like potential by changing of the dependent vari- able $u(\rho)=\frac{1}{\sqrt{\rho}} G(\rho)$, turn to as below:

$\frac{d^{2} u(\rho)}{d \rho^{2}}-\frac{1}{\left(1+\frac{\rho^{2}}{4 R^{2}}\right)^{2}} \sum_{j=-2}^{2} \bar{\delta}_{j} \rho^{j} u(\rho)=0$

where

$$
\begin{aligned}
\bar{\delta}_{-2}= & \left(\frac{m^{2}}{\alpha^{2}}-V_{3}^{2}\right)+\frac{3}{4}, \\
\bar{\delta}_{-1}= & 2\left(M+V_{1}-V_{2}+2 V_{3} \mu\right) V_{3}, \\
\bar{\delta}_{0}= & \rho^{2}+\mathcal{E}^{2}-p_{z}^{2}-\frac{2 m \mathcal{E} \Omega}{\alpha}-\frac{m^{2}}{2 \alpha^{2} R^{2}} \\
& -\left(M+V_{1}-V_{2}+2 V_{3} \mu\right)^{2}-2 V_{2} V_{3} \mu . \\
\bar{\delta}_{1}= & -2 V_{2} \mu\left(M+V_{1}-V_{2}+2 V_{3} \mu\right), \\
\bar{\delta}_{2}= & \frac{1}{16 R^{4}}\left(\frac{m}{\alpha}+4 \Omega R^{2} \mathcal{E}-V_{2}^{2} \mu^{2}\right) .
\end{aligned}
$$

Now, by supposing $V_{2}=V_{3}=0$ and using the relation $\rho=2 R \cos \theta$ onto Eq. (19), then, defining $y=\cos \theta$ and $\zeta=1-y^{2}$, we have

$$
\begin{aligned}
& \frac{d^{2} u(\zeta)}{d \zeta^{2}}-\frac{(1-2 \zeta)}{\zeta(1-\zeta)} \frac{d u(\zeta)}{d \zeta} \\
& +\frac{1}{\zeta^{2}(1-\zeta)^{2}}\left[-\left(\frac{\bar{\delta}_{2}}{4}+\frac{\bar{\delta}_{-2}}{4}-R^{2} \bar{\delta}_{0}\right) \zeta^{2}\right. \\
& \left.\quad-\left(R^{2} \bar{\delta}_{0}-\frac{\bar{\delta}_{-2}}{2}\right) \zeta-\frac{\bar{\delta}_{-2}}{4}\right] u(\zeta)=0
\end{aligned}
$$

By using the generalized NU method [57], the energy levels and wave functions are obtained as

$$
\begin{gathered}
n^{2}+\left[-1+2+2\left(\sqrt{-\left(\frac{\bar{\delta}_{2}}{4}+\frac{\bar{\delta}_{-2}}{4}-R^{2} \bar{\delta}_{0}\right)}-\sqrt{\left.\frac{\bar{\delta}_{-2}}{4}\right)}\right] n\right. \\
-\sqrt{\frac{\bar{\delta}_{-2}}{4}\left(1+2 \sqrt{\frac{\bar{\delta}_{-2}}{4}}-2 \sqrt{-\left(\frac{\bar{\delta}_{2}}{4}+\frac{\bar{\delta}_{-2}}{4}-R^{2} \bar{\delta}_{0}\right)}\right)} \\
+\sqrt{-\left(\frac{\bar{\delta}_{2}}{4}+\frac{\bar{\delta}_{-2}}{4}-R^{2} \bar{\delta}_{0}\right)}+\left(R^{2} \bar{\delta}_{0}-\frac{\bar{\delta}_{-2}}{2}\right)=0
\end{gathered}
$$

and

$u(\zeta)=\zeta^{\sqrt{\frac{\bar{\delta}_{-2}}{4}}}(1-\zeta)^{\sqrt{c_{9}}} P_{n}^{\left(-2 \sqrt{\frac{\bar{\delta}_{-2}}{4}}, 2 \sqrt{c_{9}}\right)}(1-2 \zeta)$

respectively, where

$c_{9}=\left(R^{2} \bar{\delta}_{0}-\frac{\bar{\delta}_{-2}}{2}\right)+\frac{\bar{\delta}_{-2}}{4}+\left(\frac{\bar{\delta}_{2}}{4}+\frac{\bar{\delta}_{-2}}{4}-R^{2} \bar{\delta}_{0}\right)$. 


\subsection{Scalar particle in hyperbolic coordinates}

Now, we investigate the limit of Eq. (1) where we may obtain the hyperbolic solution for $l^{2}>0$ in Eq. (1). Therefore, the metric tensor may be given in the presence of the cosmic string as below [51]:

$$
\begin{aligned}
d s^{2}= & \frac{1}{\left(1-l^{2} \rho^{2}\right)^{2}}\left(d \rho^{2}+\alpha^{2} \rho^{2} d \varphi^{2}\right) \\
& -\left(d t+\frac{\alpha \Omega \rho^{2}}{1-l^{2} \rho^{2}} d \varphi\right)^{2}+d z^{2} .
\end{aligned}
$$

In this step, we can write the radial equation form of the Klein-Gordon equation in this space-time as below:

$$
\frac{d^{2} u(\rho)}{d \rho^{2}}-\frac{1}{\left(l^{2} \rho^{2}-1\right)^{2}} \sum_{j=-2}^{2} \bar{\gamma}_{j} \rho^{j} u(\rho)=0,
$$

where

$$
\begin{aligned}
\bar{\gamma}_{-2}= & \left(\frac{m^{2}}{\alpha^{2}}-V_{3}^{2}\right)+\frac{3}{4}, \\
\bar{\gamma}_{-1}= & -2 V_{3}\left(M+V_{1}-V_{2}+2 V_{3} \mu\right), \\
\bar{\gamma}_{0}= & \rho^{2}-\mathcal{E}^{2}+p_{z}^{2}-\frac{2 l^{2} m^{2}}{\alpha^{2}}+\frac{2 m \mathcal{E} \Omega}{\alpha}-\frac{m^{2}}{2 \alpha^{2} R^{2}} \\
& +\left(M+V_{1}-V_{2}+2 V_{3} \mu\right)^{2}-2 V_{2} V_{3} \mu . \\
\bar{\gamma}_{1}= & +2 V_{2} \mu\left(M+V_{1}-V_{2}+2 V_{3} \mu\right), \\
\bar{\gamma}_{2}= & \left(\frac{m l^{2}}{\alpha}+\Omega \mathcal{E}+V_{2}^{2} \mu^{2}\right) .
\end{aligned}
$$

Now, by assuming $V_{2}=V_{3}=0$ and applying the transformation as $\rho=\frac{1}{l} \tanh (l \theta)$ onto Eq. (26), then, defining $y=\cosh (l \theta)$ and $\zeta=y^{2}-1$, we have

$$
\begin{aligned}
& \frac{d^{2} u(\zeta)}{d \zeta^{2}}+\frac{(1+2 \zeta)}{\zeta(1+\zeta)} \frac{d u(\zeta)}{d \zeta} \\
& +\frac{1}{\zeta^{2}(1+\zeta)^{2}}\left[-\left(\frac{\bar{\gamma}_{2}}{4}+\frac{\bar{\gamma}-2}{4}-R^{2} \bar{\gamma}_{0}\right) \zeta^{2}\right. \\
& \left.+\left(-R^{2} \bar{\gamma}_{0}+\frac{\bar{\gamma}-2}{2}\right) \zeta-\frac{\bar{\gamma}-2}{4}\right] u(\zeta)=0 .
\end{aligned}
$$

By using the generalized NU method [57] as

$$
\begin{aligned}
& n^{2}+\left[3+2\left(\sqrt{-\frac{\bar{\gamma}_{2}}{4}+\frac{\bar{\gamma}-2}{2}}-\sqrt{\frac{\bar{\gamma}-2}{4}}\right)\right] n \\
& +\left(\sqrt{\frac{\bar{\gamma}-2}{4}}-\sqrt{-\frac{\bar{\gamma}_{2}}{4}+\frac{\bar{\gamma}-2}{2}}-\right)\left(1+2 \sqrt{\frac{\bar{\gamma}-2}{4}}\right) \\
& +\left(R^{2} \bar{\gamma}_{0}-\frac{\bar{\gamma}-2}{2}\right)=0 .
\end{aligned}
$$

and

$$
u(\zeta)=\zeta^{\sqrt{\frac{\bar{\gamma}_{-2}}{4}}}(1+\zeta)^{-\sqrt{-\frac{\bar{\gamma}_{2}}{4}+\frac{\bar{\gamma}_{-2}}{2}}}
$$

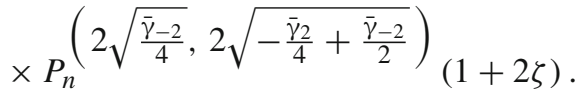

\section{Discussion and results}

In Gödel-type space-time, the solutions of the corresponding Klein-Gordon equation present us that the behavior of the scalar particle is affected by in the presence of a topological defect. However, this equation was solved in flat, spherical and hyperbolic spaces under the various interactions with more details.

Although, the expressions for the energy of the scalar particle in the three cases (flat, spherical and hyperbolic spaces) tell us that effect of the topological defect is to increase the energy levels under the Yukawa-like confinement. But, we only plotted the energy levels versus the vorticity parameter $\Omega$ and the parameter of $\alpha$ as Figs. 1 and 2 for the case of Som-Raychaudhuri space-time, respectively. Also, we investigated the limit of Eq. (1) for cases $l^{2}>0$ and $l^{2}<0$ in Eq. (1) with assuming $V_{2}=V_{3}=0$ in the Yukawa-like confinement for Eqs. (19) and (26) due to simplification the solution of our problems.

In Fig. 1, the energy levels have been plotted versus the vorticity parameter $\Omega$ with $n=1,2,3(l=0)$ and $V_{1}=0.8, V_{2}=0.4, V_{3}=0.02, \mu=0.2, \alpha=0.5, p_{z}=$ 1 and $M=2$. Figure 1 shows that the energy of the scalar particle decreases with increasing the vorticity parameter $\Omega$ as linearly at different the quantum numbers. In fact, the level energies begin to decrease as approximately exponentially, but, with increasing $l$, at the beginning, it decreases with the increasing the vorticity parameter $\Omega$, but then it reaches a minimum at around $\Omega \approx 0.1$, then it increases with the increasing the vorticity parameter $\Omega$ as fast. We notice that, for a fixed value of the vorticity parameter $\Omega$, the energy of the scalar particle increases, in Fig. 1, when the quantum numbers are increasing.

Also, In Fig. 2, the energy levels of the scalar particle have been plotted versus the parameter $\alpha$ with $l=-1,0,1$ and $V_{1}=0.8, V_{2}=0.4, V_{3}=0.02, \mu=0.2, \Omega=0.05, p_{z}=$ $1, n=2$ and $M=2$. We observe that the energy of system decreases with increasing the parameter $\alpha$ as exponentially for $l=1$. Also, in $l=0$, the level energies are as constant, but, with $l=-1$, the level energies begin to increase as exponentially with the increasing the parameter $\alpha$.

\section{Conclusion}

In this paper, we considered a Gödel-type space-time for any $l$ in the presence of a topological defect, and then analyzed the Yukawa-like confinement of a relativistic scalar particle. The corresponding wave functions were written in 
terms of the Hessenberg determinant by using the generalized series method. Moreover, the similarities between our results and the Landau levels in this spacetime background is beneficial and the Landau levels in condensed matter systems of interacting particles can be drown out [23]. In addition, Quarkonia-type systems can be described by confining potentials Model. Therefore, studying the Yukawa potential in the Som-Raychaudhuri spacetime in the presence of a topological defect is an advantage and the results of the present study can be used to investigate heavy quarkonia within a strong magnetic field.

Acknowledgements The authors would like to thank the kind referee(s) for the positive suggestions which have greatly improved the present text.

Open Access This article is distributed under the terms of the Creative Commons Attribution 4.0 International License (http://creativecomm ons.org/licenses/by/4.0/), which permits unrestricted use, distribution, and reproduction in any medium, provided you give appropriate credit to the original author(s) and the source, provide a link to the Creative Commons license, and indicate if changes were made. Funded by SCOAP ${ }^{3}$.

\section{References}

1. M. Barriola, A. Vilenki, Phys. Rev. Lett. 63, 341 (1989)

2. T.W. Kibble, J. Phys. A 19, 1387 (1976)

3. B. Linet, Gen. Relativ. Gravit. 17, 1109 (1985)

4. W.A. Hiscock, Phys. Rev. D 31, 3288 (1985)

5. G.de A. Marques, V.B. Bezerra, Phys. Rev. D 66, 105011 (2002)

6. C. Furtado, F. Moraes, Phys. Lett. A 188, 394 (1994)

7. N. Soheibi, M. Hamzavi, M. Eshghi, S.M. Ikhdair, Eur. Phys. J. B 90, 212 (2017)

8. F. Meng, A.M. Stephan, A. Forticaux, S. Jin, Acc. Chem. Res. 46, $1616(2013)$

9. G.de A. Marques, V.B. Bezerra, Class. Quantum Gravity 19, 985 (2002)

10. C. Furtado, F. Moraes, Phys. Lett. A 195, 90 (1994)

11. G.de A. Marques, C. Furtado, V.B. Bezerra, F.J. Moraes, J. Phys. A Math. Gen. 31, 5945 (2001)

12. J.L.A. Coelho, R.L.P.G. Amaral, J. Phys. A Math. Gen. 35, 5255 (2002)

13. E.R. Figueiredo Medeiros, E.R. Bezerra de Mello, Eur. Phys. J. C 72, 2051 (2012)

14. J. Carvalho, A.M.M. de Carvalho, C. Furtado, Eur. Phys. J. C 74, 2935 (2014)

15. L. Parker, Phys. Rev. Lett. 44, 1559 (1980)

16. L. Parker, L.O. Pimentel, Phys. Rev. D 25, 3180 (1982)

17. S. Moradi, E. Aboualizadeh, Gen. Relativ. Gravit. 42, 435 (2010)

18. K. Gödel, Rev. Mod. Phys. 21, 447 (1949)

19. D. Malament, in Proceedings of the Biennial Meeting of the Philosophy of Science Association, vol. II, ed. by P.D. Asquith, P. Kitcher (Chicago: University of Chicago Press, 1985), p. 91

20. P.J. Nahin, Time Machines (Springer, New York, 1999)

21. I. Ozsvath, E. Schucking, Am. J. Phys. 71, 801 (2003)

22. D. Bacon, Phys. Rev. A 70, 032309 (2004)
23. D. Deutsch, Phys. Rev. D 44, 3197 (1991)

24. J.D. Barrow, M.P. Dabrowski, Phys. Rev. D 58, 103502 (1998)

25. P. Kanti, C.E. Vayonakis, Phys. Rev. D 60, 103519 (1999)

26. H. Takayanagi, JHEP 12, 011 (2003)

27. D. Brace, JHEP 12, 021 (2003)

28. T. Harmark, T. Takayanagi, Nucl. Phys. B 662, 3 (2003)

29. S. Hawking, Phys. Rev. D 46, 603 (1992)

30. M. Reboucas, J. Tiomno, Phys. Rev. D 28, 1251 (1983)

31. M. Rebouças, M. Aman, A.F.F. Teixeira, J. Math. Phys. 27, 1370 (1985)

32. John D. Barrow, Christos G. Tsagas, Class. Quantum Gravity 21, $1773(2004)$

33. T. Clifton, J. Barrow, Phys. Rev. D 72, 123003 (2005)

34. E. Cavalcante, J. Carvalho, C. Furtado, Eur. Phys. J. Plus 131, 288 (2016)

35. G.Q. Garcia, J.R.de S. Oliveira, K. Bakke, C. Furtado, Eur. Phys. J. Plus 132, 123 (2017)

36. G.Q. Garcia, E. Cavalcante, A.M.M. de Carvalho, C. Furtado, Eur. Phys. J. Plus 132, 183 (2017)

37. G.Q. Garcia, J.R.de S. Oliveira, C. Furtado, Int. J. Mod. Phys. D 27, 1850027 (2018)

38. I. Ozsvath, J. Math. Phys. 6, 590 (1965)

39. I. Ozsvath, J. Math. Phys. 11, 2871 (1970)

40. J. Carvalho, A.M.M. de Carvalho, E. Cavalcante, C. Furtado, Eur. Phys. J. C 76, 365 (2016)

41. F.M. Paiva, M.J. Reboucas, A.F.F. Teixeira, Phys. Lett. A 126(3), 168 (1987)

42. Z. Wang, Z.-W. Longa, C.-Y. Long, M.-L. Wu, Eur. Phys. J. Plus 130, $36(2015)$

43. R.L.L. Vitória, C. Furtadoa, K. Bakke, Eur. Phys. J. C 78, 44 (2018)

44. D.V. Gal'tsov, E. Masar, Class. Quantum Gravity 6, 1313 (1989)

45. M. Aryal, L.H. Ford, A. Vilenkin, Phys. Rev. D 34, 2263 (1986)

46. M.G. Germano, V.B. Bezerra, E.R. Bezerra de Mello, Class. Quantum Gravity 13, 2663 (1996)

47. E.R. Bezerra de Mello, A.A. Saharian, J. Phys. A Math. Theor. 45, 115402 (2012)

48. H. Yukawa, On the interaction of elementary particles. Proc. Phys. Math. Soc. Jpn. 17, 48 (1935)

49. H. Taseli, Int. J. Quantum Chem. 63, 949 (1997)

50. M.W. Kermode, M.L.J. Allen, J.P. Mctavish, A. Kervell, J. Phys. G Nucl. Part. Phys. 10, 773 (1984)

51. N.R. Napolitano, S. Capozziello, A.J. Romanowsky, M. Capaccioli, C. Tortora, Astrophys. J. 748, 87 (2012)

52. S.M. Ikhdair, M. Hamzavi, Z. Naturforsch. 68a, 715 (2013)

53. M.M. Som, A.K. Raychaudhuri, Proc. R. Soc. A 304, 81 (1968)

54. M. Eshghi, H. Mehraban, Azar I. Ahmadi, Phys. E 94, 106 (2017)

55. M. Eshghi, H. Mehraban, Azar I. Ahmadi, Eur. Phys. J. Plus 132, 477 (2017)

56. K. Kaygisız, A. Sahin, Gen. Math. Notes 9(2), 32 (2012)

57. C. Tezcan, R. Sever, Int. J. Theor. Phys. 48, 337 (2009) 